\title{
MANEJO DE LA MEDICACIÓN EN EL ADULTO MAYOR AL ALTA HOSPITALARIA
}

\section{MEDICATION MANAGEMENT IN THE ELDERLY AT DISCHARGE}

\author{
María Guadalupe Moreno Monsiváis* \\ Leticia Garza FernándeZ \\ Ma. Guadalupe InTERIAL GuZMÁN ${ }^{* * *}$
}

\begin{abstract}
RESUMEN
Objetivo: Identificar el manejo de la medicación en el adulto mayor al alta hospitalaria de una institución privada de Monterrey, N.L. México y su asociación con las características individuales edad, escolaridad, sexo y polifarmacia. Material y método: Diseño descriptivo correlacional, muestra de 162 adultos mayores seleccionados a través de un muestreo probabilístico sistemático. Se utilizó el instrumento MedMaIDE para identificar deficiencias en el manejo de la medicación en el adulto mayor. Resultados: La media de medicamentos prescritos fue de 7 ( $D E=3.33)$; un $26.5 \%$ de los participantes presentaron al menos una deficiencia en el manejo de su medicación. Los resultados fueron similares para hombres y mujeres, las deficiencias se asociaron con la edad $(r s=0.240 ; \mathrm{p}=0.001)$, escolaridad $(r s=-0.158 ; \mathrm{p}=0.04)$ y polifarmacia $(r s=0.370 ; \mathrm{p}=0.001)$. Conclusión: Se encontró un déficit en la medicación de los adultos mayores. Estos resultados deben ser considerados en el desarrollo de intervenciones dirigidas a garantizar que el plan de alta incluya educación para el manejo de la medicación en el hogar.
\end{abstract}

Palabras clave: Conocimiento de la medicación por el paciente, adulto mayor, polifarmacia, alta hospitalaria.

\begin{abstract}
Objective: Identify the medication management in the elderly at discharge from a private institution of Monterrey; NL México and the association with age, education, sex and polypharmacy individual characteristics. Method: A descriptive, correlational design was used with a sample of 162 older adults selected through a systematic probability sampling. The MedMaIDE instrument was used. Results: The average number of drugs prescribed was $7(S D=3.33), 26.5 \%$ of participants had at least one deficiency in the management of their medication. The results were similar for men and women, the deficiencies were associated with age $(r s=0.240 ; \mathrm{p}$ $=0.001)$, education $(r s=-0.158 ; \mathrm{p}=0.04)$, and polypharmacy $(r s=0.370 ; \mathrm{p}=0.001)$. Conclusion: We found a deficit in the elderly medication. These results should be considered in the development interventions to ensure that the discharge plan includes education for medication management at home.
\end{abstract}

Key words: Patient medication knowledge, elderly, polypharmacy, patient discharge.

Fecha recepción: 12/07/13 Fecha aceptación: 03/11/13

\footnotetext{
* Licenciada y Máster en Enfermería. Dra. en Administración. Profesor Facultad de Enfermería, Universidad Autónoma de Nuevo León. Monterrey, N.L. México. Gonzalitos 1500, Nte. Col. Mitras Centro, Monterrey, Nuevo León, México. Tel. (0181)83-48-18-47 Fax 834811 07. E-mail: mgmoreno@hotmail.com

** Licenciada y Máster en Ciencias de Enfermería. Hospital San José del TecSalud Monterrey, N.L. México. E-mail: lgarza@ tecsalud.mx

${ }^{* * *}$ Licenciada y Máster en Ciencias de Enfermería. Profesor Facultad de Enfermería, Universidad Autónoma de Nuevo León. Monterrey, N.L. México. E-mail: mginterial@hotmail.com
} 


\section{INTRODUCCIÓN}

Uno de los procesos prioritarios en el marco de la seguridad del paciente es el manejo de la medicación $(1,2)$. Es fundamental que todo sistema de salud busque estrategias para garantizar un manejo adecuado de la medicación en los pacientes hospitalizados que van a ser dados de alta. Existe evidencia que la falta de un manejo adecuado de la medicación tiene múltiples repercusiones, por lo que es elemental asegurarse que los pacientes poseen el conocimiento acerca de la medicación que tomarán en su casa; cómo deberán tomar los medicamentos y cómo los obtendrán. La Organización Mundial de la Salud (3) afirma que $50 \%$ de los pacientes toman de manera incorrecta los medicamentos prescritos, lo que genera mayor riesgo de hospitalización, incremento en la presencia de reacciones adversas a los medicamentos, así como en el índice de morbilidad y mortalidad. Este déficit en el manejo de la medicación se incrementa conforme avanza la edad del paciente (4).

Los adultos mayores son un grupo de riesgo en el manejo de la medicación por el esquema o situación de salud que los caracteriza como son las pluripatologías y predominio de padecimientos crónicos (5). Esta situación de salud demanda tratamientos prolongados y variados, lo que se traduce en utilización de polifarmacia; de acuerdo a lo que se ha reportado en la literatura los adultos mayores tienen prescritos de uno a once medicamentos. Sin embargo, otros reportes señalan hasta 18 medicamentos prescritos $(1,5-9)$.

Otro factor importante que incrementa el riesgo en el manejo de la medicación en este grupo poblacional es el proceso de deterioro progresivo que acontece durante el envejecimiento y afecta la función de múltiples órganos y sistemas, lo que genera diferentes alteraciones como déficit visual, auditivo, en la capacidad física, en el estado cognitivo, cambios en los estilos de vida, algún grado de dependencia, entre otras (10). Todas estas alteraciones tienen repercusiones en la autoadministración de medicamentos ya sea por dosis inadecuada (mayor o menor a la prescrita), incumplimiento en los horarios, errores en el procedimiento de administración, entre otros $(5,8)$.

La literatura internacional reporta que existen factores individuales que pueden predecir el riesgo de manejo inadecuado en la medicación. Se ha encontrado que el sexo femenino se considera un grupo más vulnerable por presentar una mayor prevalencia de polifarmacia, lo cual se asocia al mayor predominio de enfermedades de tipo crónico $(7,8)$. Sin embargo, los hombres reportan menor adherencia al tratamiento farmacológico; este resultado mejora si se cuenta con una persona que colabore en su manejo de la medicación (5). La baja escolaridad es otro factor considerado de riesgo, esto debido a la falta de comprensión en el tratamiento, lo que genera mayor presencia de errores $(6,8)$.

El equipo de salud juega un rol fundamental en el manejo de la medicación. Los estándares de cuidado al paciente destacan como indicador de calidad, la educación y entrenamiento para el manejo de la medicación por parte del personal de salud en todo paciente, miembros de la familia y personas significativas, de tal forma que se asegure que poseen el conocimiento específico que les permita desarrollar las habilidades necesarias para un manejo adecuado de la medicación $(2,11)$. En este sentido, el alta hospitalaria se considera un momento crítico en el que el personal de salud debe proporcionar la educación para el manejo de la medicación en el hogar y avalar que el paciente a su egreso tiene conocimiento de los medicamentos indicados, cómo administrarlos y cómo proveerse de ellos (11-14).

De acuerdo a Orwig et al. (15), el manejo de la medicación por parte del paciente es un proceso complejo que está integrado por tres dominios que permiten asegurar su 
cumplimiento, éstos son: conocimiento de la medicación, conocer cómo los debe tomar y conocer cómo los puede obtener y el acceso a ellos. El paciente necesita conocer la indicación, cómo y cuándo se deben administrar, la duración del tratamiento y cómo obtener sus medicamentos para asegurar el cuidado integral y la continuidad del cuidado en el hogar, además como parte integral del adecuado manejo de la medicación es importante confirmar que el paciente conoce cómo almacenar de manera adecuada los medicamentos (luz, temperatura y humedad).

La evidencia de vulnerabilidad en los adultos mayores aunado al incremento de población en este grupo de edad, enfatiza la responsabilidad del equipo de salud de garantizar la seguridad y eficacia del tratamiento farmacológico. Esto constituye un área de oportunidad para que el personal de enfermería coordine sus acciones con el personal médico, farmacéutico, familiares o cuidadores y con el propio adulto mayor involucrado en el tratamiento y se asegure que en el plan de alta se proporcione una educación efectiva acerca del manejo farmacológico y se valide el conocimiento, las habilidades para su manejo, así como el acceso a la medicación indicada.

En consideración a lo anterior, el propósito del estudio fue identificar el manejo de la medicación en el adulto mayor al alta hospitalaria de una institución privada y su asociación con las características individuales: edad, escolaridad, sexo, y polifarmacia.

\section{MATERIAL Y MÉTODO}

El presente estudio fue realizado en un hospital privado de tercer nivel de atención ubicado en el área metropolitana de Monterrey, N.L. México. El diseño fue descriptivo y correlacional (16). La población estuvo conformada por pacientes de 60 años a más, que fueron dados de alta de los servicios de medicina interna y posquirúrgicos con medicación prescrita al alta hospitalaria y que autoadministraban su medicación. El tipo de muestreo para la selección de pacientes fue probabilístico sistemático de uno en dos con inicio aleatorio.

El tamaño de la muestra se estimó a través del paquete nQuery Advisor versión 4.0 (17) para un análisis de correlación. Se consideró un nivel de significancia de 0.05 , una correlación esperada de 0.40 (de acuerdo a lo reportado en estudios previos), una potencia de $90 \%$. El tamaño de la muestra total fue de 162 adultos mayores participantes.

El procedimiento consistió en visitar los servicios de hospitalización de medicina interna y posquirúrgicos de las áreas de cirugía general, ginecología y traumatología en un horario de 9 a 10 de la mañana, para identificar posibles altas hospitalarias de adultos mayores, se acudió con los pacientes seleccionados aleatoriamente y se verificó que tuvieran medicación prescrita al alta hospitalaria y que autoadministraran su medicación. A los que reunieron estos criterios se les explicó el objetivo del estudio, se solicitó su participación voluntaria y a los que aceptaron se les pidió la firma de la Carta de Consentimiento Informado.

Los datos se recolectaron a través de una Cédula de Identificación en la cual se registraron las características del paciente relacionadas con edad, sexo, diagnóstico, escolaridad, si vive solo o acompañado. Se incluyó además un apartado en el que se obtuvo información relacionada con la prescripción de medicamentos para el alta y el tratamiento farmacológico que el paciente tomaba de manera permanente; se registró el nombre, dosis, vía de administración y horario, esta información se utilizó para la conciliación de la medicación; adicionalmente se preguntó si recibe ayuda para administrar alguno de los medicamentos y quién le proporcionó la orientación para el manejo de su medicación en el hogar. Se continuó con una entrevista al paciente para el llenado del cuestionario 
MedMaiDE de Orwig et al. (15), el cual permite identificar deficiencias en el manejo de la medicación en el adulto mayor. Consta de 19 reactivos divididos en tres dominios, el primero identifica lo que una persona debe conocer acerca de su medicación, el segundo está dirigido a identificar si el paciente sabe como tomar sus medicamentos y demostrar las habilidades para ello. El tercero determina si el paciente conoce como obtener o tener acceso a surtir sus medicamentos.

El instrumento tiene opciones de respuesta dicotómica (Sí/No). Sólo 13 reactivos tienen valor, por lo tanto el total de calificación para deficiencia en el manejo de la medicación fluctúa desde cero puntos como calificación mínima, identificada como no deficiencia, hasta 13 puntos, calificación máxima de deficiencia en el manejo de la medicación. La consistencia interna reportada a través del Coeficiente Alpha de Cronbach es de 0.71, para el presente estudio se obtuvo un resultado de 0.75 .

El estudio se realizó con apego a lo dispuesto en la Ley General de Salud en Materia de Investigación para la Salud y fue aprobado por las Comisiones de Ética e Investigación de la Facultad de Enfermería de la Universidad Autónoma de Nuevo León (18). Se respetó la dignidad y la protección de los derechos de los participantes, la participación voluntaria y la libertad de retirarse en el momento que lo desearan. Se contó con el consentimiento informado por escrito de los adultos mayores posterior a recibir la explicación clara acerca del estudio y en qué consistiría su participación.

Se utilizó estadística descriptiva para determinar las características de los adultos mayores y el manejo de su medicación. Para el análisis estadístico inferencial se consideró la normalidad de los datos a través de la prueba de Kolmogorov-Smirnov, únicamente la edad mostró distribución normal por lo que se utilizó el Coeficiente de Correlación de Spearman para determinar la relación en- tre las deficiencias en el manejo de la medicación con la edad, escolaridad y polifarmacia. La diferencia en el manejo de la medicación de acuerdo al sexo se obtuvo con la prueba de $\mathrm{X}^{2}$.

\section{RESULTADOS}

Los adultos mayores participantes del estudio, reportaron una edad promedio de 70 años $(D E=7.31)$; la escolaridad se ubicó en 12 años $(D E=4.39)$, con un valor mínimo de 0 hasta 20 años de estudio. Al analizar la escolaridad de acuerdo al sexo, en el masculino predominó el nivel más alto, mientras que el $68 \%$ de los hombres son profesionistas, sólo un $24 \%$ de las mujeres alcanzan este nivel de estudio.

La proporción de participantes del sexo masculino fue similar a la del femenino (48.1 vs $51.9 \%$ respectivamente). En cuanto a la especialidad médica a la que pertenece el diagnóstico de ingreso de los pacientes, un $62.3 \%$ corresponde a medicina interna y el resto a posquirúrgicos. La mayor proporción de adultos mayores refirió vivir con su cónyuge o con cónyuge e hijos, sólo el $4.3 \%$ de ellos vive solo y el $3.7 \%$ con empleados domésticos.

\section{Medicación del adulto mayor}

En la Tabla 1 se muestra la información referente a los medicamentos que el paciente reportó tomar en forma habitual a su ingreso hospitalario, los prescritos al alta, así como el total de medicamentos que tomará a su alta hospitalaria. Los pacientes toman en promedio cuatro medicamentos a su ingreso hospitalario $(D E=2.88)$ y la cantidad que les prescriben al alta hospitalaria es similar. Al conciliar la medicación habitual y la prescrita para el alta se registró un total de siete medicamentos en promedio $(D E=3.33)$. 
Tabla 1. Número de medicamentos del paciente al ingreso, los prescritos al alta y total.

\begin{tabular}{lccccc}
\hline \multicolumn{1}{c}{ Medicamentos } & Media & Mediana & $D E$ & Mín. & Max. \\
\hline Habituales al ingreso & 4.45 & 4 & 2.88 & 0 & 16 \\
Prescritos al alta hospitalaria & 4.37 & 4 & 2.58 & 0 & 14 \\
Total de medicamentos (conciliación) & 7.59 & 7 & 3.33 & 1 & 23 \\
\hline
\end{tabular}

Fuente: Cédula de Datos del Paciente $n=162$

\section{Manejo de la Medicación al Alta Hospitalaria}

Los resultados del primer dominio del manejo de la medicación referente al conocimiento que el paciente tiene acerca de la medicación que manejará en casa al alta hospitalaria, reflejan que las principales deficiencias se ubicaron en la dificultad para señalar para qué toma los medicamentos con un $21 \%$, seguido de la dificultad para mencionar la dosis correcta (17.9\%). Un escaso porcentaje reportó dificultad para identificar la vía de administración (Tabla 2). El 26.5\% reportó que el cónyuge es la persona que les ayuda en la administración de los medicamentos; al analizar estos datos de acuerdo al sexo, se encontró que una mayor proporción de hombres reciben ayuda en la medicación (29.5\% de hombres / 3.6\% de mujeres).

Tabla 2. Conocimiento acerca de la medicación.

\begin{tabular}{lccrc}
\hline \multicolumn{1}{c}{ Conocimiento } & \multicolumn{2}{c}{ Sí } & \multicolumn{2}{c}{ No } \\
\hline Menciona los medicamentos que tomará en casa & 139 & 85.8 & 23 & 14.2 \\
Menciona el horario en que los tomará & 137 & 84.6 & 25 & 15.4 \\
Menciona la vía de administración & 160 & 98.8 & 2 & 1.2 \\
Conoce para qué está tomando cada medicamento & 128 & 79.0 & 34 & 21.0 \\
Menciona la cantidad de cada medicamento & 133 & 82.1 & 29 & 17.9 \\
\hline
\end{tabular}

Fuente: Instrumento MedMaIDE $n=162$

En cuanto al segundo dominio referente al conocimiento del paciente acerca de cómo tomar sus medicamentos, las deficiencias se atribuyen principalmente a mencionar el número de pastillas para la dosis indicada (3.7\%), seguido de la dificultad para mencionar cómo los administra (2.5\%). En este dominio se registró cómo el paciente guarda los medicamentos, se identificó que el 15\% de ellos no almacena los medicamentos de forma adecuada (humedad, luz, temperatura).
En el tercer dominio referente al conocimiento acerca de cómo obtener sus medicamentos, se observó que el total de participantes conoce cómo obtenerlos; una escasa proporción desconoce si debe resurtirlos $(2.5 \%)$ y sólo un paciente tenía dudas acerca de a quién contactar para resurtirlos $(0.6 \%)$. En este último apartado se cuestionó al paciente si revisa la medicación al resurtirla, se encontró que el $30.9 \%$ de los pacientes no revisa la caducidad y $11.7 \%$ no revisa que el medicamento surtido corresponda a la pre- 
sentación solicitada.

Posterior al análisis individual de cada uno de los dominios, se obtuvo la proporción de adultos mayores que presentaron al menos una deficiencia en alguno de los dominios y en forma global en el manejo de la medicación. En la Tabla 3 se observa que el
26.5\% de los participantes presentaron al menos una deficiencia en cuanto al conocimiento acerca de su medicación. La menor proporción de deficiencias correspondió al conocimiento que tiene el paciente acerca de cómo obtener su medicación.

Tabla 3. Manejo de la medicación por dominios.

\begin{tabular}{lcc}
\hline \multicolumn{1}{c}{ Dominio } & \% sin déficit & \% con déficit \\
\hline Conocimiento acerca de la medicación & 73.5 & 26.5 \\
Conoce cómo tomar sus medicamentos & 92.6 & 7.4 \\
Conoce cómo debe obtener sus medicamentos & 96.9 & 3.1 \\
Manejo de la medicación & 72.8 & 27.2 \\
\hline
\end{tabular}

Fuente: Instrumento MedMaIDE $n=162$

Se analizó el manejo de la medicación de acuerdo al sexo; sin embargo, no se encontró diferencia significativa $(p>0.05)$. Respecto a la persona que les otorgó la educación para el manejo de la medicación al alta hospitalaria; la mayoría de los pacientes refirió a su médico tratante $(75.3 \%)$, una menor proporción señaló a la enfermera (17.9\%), el resto reportó recibir la educación de ambos profesionales.

\section{Relación del manejo de la medicación con edad, escolaridad y polifarmacia}

En la Tabla 4 se observan los resultados del Coeficiente de Correlación de Spearman $(r s)$ para determinar la relación entre las deficiencias en el manejo de la medicación con las características del paciente: edad, escolaridad y polifarmacia. La edad se asoció con las deficiencias en el manejo de la medica-

Tabla 4. Correlación de deficiencias en el manejo de la medicación con edad, escolaridad y polifarmacia.

\begin{tabular}{lcccc}
\hline Características & $\begin{array}{c}\text { Deficiencia } \\
\text { conocimiento }\end{array}$ & $\begin{array}{c}\text { Deficiencia cómo } \\
\text { toma }\end{array}$ & $\begin{array}{c}\text { Deficiencia cómo } \\
\text { obtiene }\end{array}$ & $\begin{array}{c}\text { Deficiencia } \\
\text { Total }\end{array}$ \\
\hline Edad & $0.222^{\star * *}$ & $0.250^{\star * *}$ & -0.028 & $0.240^{\star * *}$ \\
Escolaridad & -0.141 & -0.124 & -0.013 & $-0.158^{\star}$ \\
Polifarmacia & $0.383^{\star * \star}$ & $0.238^{\star * *}$ & 0.010 & $0.370^{\star * \star}$ \\
\hline
\end{tabular}

Fuente: Cédula de Datos e Instrumento MedMaIDE $n=162$

Coeficiente de Correlación de Spearman

${ }^{* *} 0.001$ Altamente significativo ${ }^{* *} 0.01$ Considerablemente significativo

${ }^{\star} 0.05$ Significativo 
ción $(r s=0.240 ; p=0.001)$. Así mismo, se relacionó positivamente con las deficiencias en el conocimiento acerca de la medicación y con el conocimiento de cómo tomarlo; sin embargo, no se asoció significativamente con el conocimiento que el paciente tiene para obtener su medicación.

Se encontró una débil relación inversa entre la deficiencia total para el manejo de la medicación y la escolaridad del paciente $(r s=-0.158 ; p=0.04)$. La polifarmacia mostró una relación significativa con el déficit en el manejo de la medicación ( $r s=0.370 ; p=$ 0.001 ). Al analizar por dominios, la polifarmacia se asoció con las deficiencias respecto al conocimiento de la medicación y las referentes a cómo tomarlo.

\section{DISCUSIÓN Y CONCLUSIÓN}

Los resultados del estudio permitieron identificar que una cuarta parte de los adultos mayores presentó al menos una deficiencia en cuanto al conocimiento de su medicación. La mayor dificultad referida por los pacientes fue el señalar para qué toma cada uno de sus medicamentos, aproximadamente una quinta parte desconoce la indicación de su medicación, es decir para qué toma su medicamento; esta proporción coincide con lo reportado por Sanches et al. (8), sin embargo difiere respecto a los hallazgos de otros autores (15), quienes señalan una mayor proporción de pacientes que no conocen la indicación de su medicación. Esta diferencia pudiera atribuirse a las características de la muestra del presente estudio, los adultos mayores participantes tienen acceso a una institución privada, pertenecen a un nivel socioeconómico medio alto y alto; y aunado a esto, reportaron un nivel alto de escolaridad, estos factores pudieran explicar los hallazgos en relación a un mayor conocimiento de la indicación de su medicación.
La literatura es consistente al señalar que el principal factor asociado a la toma incorrecta de los medicamentos es la falta de conocimiento (5). El conocer el motivo por el cual se toman los medicamentos es fundamental para un adecuado manejo de la medicación (19). A pesar de que en este estudio se encontró un mayor conocimiento respecto a lo reportado por algunos autores, como ya se señaló previamente, los hallazgos deben ser considerados como un área de oportunidad debido a que se debe de garantizar que todo paciente a su egreso hospitalario cuente con un adecuado y suficiente conocimiento para el manejo de la medicación en el hogar. Así mismo, es relevante un mayor involucramiento por parte del personal de enfermería; como se señaló previamente, una escasa proporción participa en esta actividad, siendo que la educación al paciente y su familia constituyen un aspecto fundamental del rol de cuidado de enfermería.

Con respecto al conocimiento acerca de cómo tomar la medicación, la mayoría de los pacientes mostró capacidad suficiente para la autoadministración, una escasa proporción refirió dificultad para mencionar cuántas pastillas se requerían para la dosis prescrita por su médico, este aspecto se relaciona con la prescripción de los medicamentos que cuentan con diferentes presentaciones (dosis). El equipo de salud debe asegurarse que los pacientes han comprendido la posología de su medicación (6), y aún, ante mínimas deficiencias identificadas, como fue el caso en el presente estudio, deben explicarse detalladamente las características de los medicamentos, incluyendo la información sobre las diferentes presentaciones farmacéuticas; con respecto a la posología es fundamental utilizar términos sencillos para mejorar el entendimiento del paciente, sobre todo en grupos vulnerables como es el caso de los adultos mayores.

En relación al conocimiento de cómo obtener los medicamentos, se encontró que una mínima proporción de adultos mayores 
reportaron deficiencias. Éstas correspondieron a la dificultad para identificar si deben resurtir su medicación, hallazgo similar a lo reportado en la literatura (14). Este dato es importante de destacar debido a que los participantes que mostraron deficiencia contaban con una prescripción al alta que no especificaba la duración del tratamiento. De acuerdo a algunos organismos responsables de la seguridad farmacológica, como la Joint Commission International (2) y la Comisión Permanente de Farmacopea de los Estados Unidos Mexicanos (20), las prescripciones de medicamentos deben regirse por políticas y procedimientos donde se especifique la duración del tratamiento (21), por lo que debe ser considerado en el momento en que los profesionales de la salud examinan el plan de alta de sus pacientes.

La literatura es consistente acerca de las diferencias en el manejo de la medicación de acuerdo al sexo $(5,8)$; sin embargo, en el presente estudio esta diferencia no fue evidente. Esto pudiera explicarse debido a las características de los participantes, los hombres señalaron recibir apoyo por parte del cónyuge para el manejo de su medicación y en su mayoría reportaron vivir acompañados. Este hallazgo concuerda con la evidencia reportada acerca de que las personas que reciben algún tipo de apoyo para el manejo de su medicación tienen mayor probabilidad de ser más adherentes a sus tratamientos farmacológicos. Otro aspecto que pudo contribuir es el nivel alto de escolaridad reportado por los participantes.

En relación a las características individuales que se asocian con las deficiencias en el manejo de la medicación, se encontró que a mayor edad se presenta mayor deficiencia para el manejo de la medicación. Esto concuerda con lo reportado en diversos estudios donde se destaca que a mayor edad se incrementa el riesgo de errores de medicación ( 1 , $5,7,8,22)$.

Se encontró que a mayor escolaridad, menor es la deficiencia en el manejo de la medi- cación. Este hallazgo difiere con los reportes de algunos autores quienes no encontraron asociación entre escolaridad y manejo de la medicación $(5,15)$. La diferencia pudiera atribuirse a que los participantes en estos estudios reportaron niveles bajos de escolaridad en su mayoría; sin embargo, en el presente estudio se pudieron identificar variantes en el nivel de formación encontrando desde niveles bajos, sobre todo en las mujeres, hasta el predominio de un nivel universitario superior. Este dato es importante de considerar, la baja escolaridad se ha asociado con mayor riesgo de errores en el manejo de la medicación por la falta de comprensión en el tratamiento. Por lo anterior, el equipo de salud debe proporcionar una educación efectiva acerca del tratamiento farmacológico y validar en los adultos mayores con baja escolaridad, que previo a su egreso hospitalario tengan claridad acerca de su medicación, cómo administrarla y cómo tener acceso a ella.

La polifarmacia fue una condición común en los adultos mayores del estudio con media de 7 medicamentos al alta y hasta 23 como máximo, muy cercano a la media de nueve reportada en la literatura $(1,5,8)$. Se encontró que a mayor polifarmacia mayor presencia de deficiencias en el manejo de la medicación, esto coincide con lo reportado por diversos autores. Esta condición ha sido ampliamente referida por los autores como predictor de riesgo en el manejo de la medicación, especialmente en el adulto mayor ( 1 , $5,8,13,19,22)$.

Se concluye que el déficit en el manejo de la medicación identificado en los adultos mayores participantes demanda la necesidad de desarrollar intervenciones efectivas orientadas a fortalecer el adecuado manejo de la medicación en los adultos mayores previo al alta hospitalaria, como una estrategia que contribuya a garantizar la seguridad en el manejo de la medicación en el hogar. Los resultados del estudio son relevantes para la gestión del cuidado por los profesionales de 
enfermería, las intervenciones educativas en relación con el manejo de la medicación en un grupo prioritario como son los adultos mayores deben considerar como aspectos relevantes su edad y escolaridad, así como el número de medicamentos prescritos, aspectos que se asocian con las deficiencias en el manejo de la medicación.

\section{REFERENCIAS}

1. Fernández LC, Barón B, Vázquez B, Martínez T, Urendes, JJ, Pujol E. Errores de medicación e incumplimiento terapéutico en ancianos polimedicados. Farm Hosp. 2006; 30(5): 280-283.

2. Joint Commission International. Estándares de Acreditación para Hospitales. 2008. Oak Brook Illinois.

3. Organización Mundial de la Salud. The safety of medicines an Essentials tool. 2006. Geneva, World Health Organization.

4. Arriagada L, Jirón M, Ruiz I. Uso de medicamentos en el adulto mayor. Rev Hosp Clin Univ Chile. 2008; 19: 309-317.

5. Castellano-Muñoz P, Miranda-Ruiz A, Sojo-González G, Perea-Milla E, GarcíaAlegría JJ, Santos-Rubio MD. Adherencia al tratamiento farmacológico en pacientes ancianos tras el alta hospitalaria. Enferm Clín. 2008; 18(3): 120-126.

6. Leal M, Abellán J, Casa MT, Martínez J. Paciente polimedicado: ¿conoce la posología de la medicación?, ¿afirma tomarla correctamente? Aten Primaria. 2004; 33(9): 451-456.

7. Reyes A, Pérez G, Martínez G. Errores en la medicación del adulto mayor en el área de salud del Policlínico Universitario, Plaza, Ciudad de La Habana. Rev Cubana Farm. 2006; 40(3): 1-11.

8. Sanches MJ, Oliveira LC, Ferrazoli AE, Santella F, Andrade CB, Goncalves JR et al. Use of medicines by the elderly in a
Family Health Program unit in Brazil. Cad. Saúde Pública. 2008; 24(7): 15451555.

9. Garrido E, García I, García J, García F, Ortega I, Bueno A. Estudio de pacientes polimedicados mayores de 65 años en un centro de asistencia primaria urbano. Rev. Calid. Asist. 2011; 26(2): 90-96.

10. González CA, Ham-Chande R. Funcionalidad y salud: una tipología del envejecimiento en México. Salud Pública Méx. 2007; 49(4): 448-458.

11. Estándares para la certificación de hospitales, 2012. Consejo de Salubridad General. Documento Oficial. http://www. inper.edu.mx/descargas/pdf/EstandaresCertificacionHospitales2012.pdf

12. Delate T, Chester EA, Pharm D, Stubbings TW, Pharm D, Barnes CA. Clinical Outcomes of a Home-Based Medication Reconciliation Program After Discharge from a Skilled Nursing Facility. Pharmacotherapy. 2008; 28(4): 444-452.

13. Hernández C, Mira A, Arroyo E, Díaz M, Andreu L, Sánchez M. Discrepancias de conciliación en el momento del alta hospitalaria en una unidad médica de corta estancia. Aten Primaria. 2008; 40(12): 597-602.

14. Midlöv P, Holmdahl L, Eriksson T, Bergkvist A, Ljungberg B, Widner $\mathrm{H}$ et al. Medication report reduces number of medication errors when elderly patients are discharged from hospital. Pharm World Sci, 2008; 30(1): 92-98.

15. Orwig D, Brandt N, Gruber-Baldini AL. Medication management assessment for older adults in the community. Gerontologist. 2006; 46(5): 661-668.

16. Burns N, Grove SK. Investigación en Enfermería (de la $3^{a}$. Ed. del original). España: Elsevier. Alba. 2004.

17. Elashoff D, Dixon J, Crede M, Fothenringham N. n'Query Advisor, Version 4.0. 2000.

18. Secretaría de Salud [SS]. Reglamento de la Ley General de Salud en Materia de In- 
vestigación para la Salud. (7a ed). México: Purrúa. 1987.

19. De Vicente L, Rodríguez E. Polifarmacia en el paciente anciano. JANO. 2007; 4044.

20. Farmacopea de los Estados Unidos Mexicanos [FEUM]. Suplemento para establecimientos dedicados a la venta y suministro de medicamentos y otros insumos para la salud ( $\left.4^{\mathrm{a}} \mathrm{ed}\right)$. Secretaría de Salud. 2010.
21. Peralta-Pedrero M, Valdivia-Ibarra F, Hernández-Manzano $M$, Medina-Beltrán G, Cordero-Guillén $M$, Baca-Zúñiga J et al. Guía de práctica clínica Prescripción farmacológica en el adulto mayor. Rev Med Inst Mex Seguro Soc. 2013; 51 (2): 228-239.

22. Shi S, Mörike K, Klotz U. The clinical implications of ageing for rational drug therapy. Eur J Clin Pharmacol. 2008; 64(2): 183-199. 